\title{
DIRTY THE WATERS: MOTHERS' EXPERIENCE OF A CHEMICAL DISASTER IN WEST VIRGINIA, USA
}

\author{
EMILY E. PARSONS \& EMILY DIAMOND \\ The Wright Institute, USA
}

\begin{abstract}
The World Health Organization estimates that 12.6 million deaths a year are attributable to unhealthy environments, while modern industry uses more than 100,000 different chemicals in their operations, most of them understudied. On 9 January 2014, a major chemical spill in Chemical Valley, West Virginia, polluted the water of 300,000 residents across nine counties of this Appalachian region of the United States. At the time of the disaster, and even now, there is very limited knowledge about the chemical in terms of health effects. Approximately 10,000 gallons of 4-Methylcyclohexanemethanol (MCHM), which is used in coal processing, began leaking from a 48,000-gallon tanker in the early morning hours. Residents were not notified of a "Do not use" order until later that evening, after which people had no potable water, and no water for sanitation. This study explores, through structured interviews, the experience of mothers, some pregnant, some with ill children, others with multiple children, in their attempts to manage during the crisis. The study focuses on their levels of trust in systems that many felt failed them throughout the course of the disaster. Communication (official and non-official), the accessibility of clean water, community resilience and coping, caring for their children, immediate and long-term health risks, and the psychological and economic impacts of this disaster will be discussed. This study focused on 35 mothers. It's the first study to explore this particular demographics' experience and their continued adjustment in the following years. The structured interview used for this project holds promise for better understanding the experience of mothers in other chemical disasters, in which parents confront limited information, the need to make difficult choices, limited resources, and the lasting echoes of those decisions. It's hoped that it can be used in other contexts to deepen our understanding of disaster management.

Keywords: chemical spill, toxic environments, 4-Methylcyclohexanemthanol, Chemical Valley, West Virginia water crisis, Elk River, MCHM, polyglycol ethers, water contamination, freedom industry, epigenetic effects, family environmental health.
\end{abstract}

\section{INTRODUCTION}

On 9 January 2014, 48,000 gallons of 4-Methylcyclohexanemethanol (MCHM) leaked out of Freedom Industries' Tank \#396 into the Elk River, contaminating an area populated by over 300,000 people spanning nine counties, and affecting the drinking water of one-sixth of West Virginia's population. MCHM is a chemical slightly soluble in water, used to clean coal, by separating the coal from rocks and debris [1]. Annually, more than hundred million tons of coal are mined from underground seams, surface mines and mountain top removal, and then processed in the state [2]. MCHM is one the chemicals used in this process, and smells somewhat like licorice. Ten-thousand gallons of MCHM seeped into the municipal water system, along with polyglycol ethers (PPH), a solvent also used to further clean coal, which is faintly yellow in colour. Together they contaminated the state's largest water treatment plant situated a mile upriver. This water treatment plant served $15 \%$ of West Virginia's population [3], [4]. The smell and the slight yellow colour were the primary warnings to the population that the water had been contaminated. Late that evening, President Obama declared a state of emergency, which would last for 50 days.

At the time, people knew very little about this chemical, which is used to "bathe" and clean coal before it's burned for use [5]. A search of the US National Library of Medicine, National Institutes of Health Library, PubMed, holds no health studies of the chemical prior 
to this event. Even today, there is not much that is known about MCHM, including whether it could cause birth defects, cancer, or epigenetic changes, or how it might work synergistically with other chemicals, or its potential to cause ecosystem damage.

The site of this disaster was in southern West Virginia, in and around the state's capital, and one of the most economically depressed areas of the United States. For nearly a century, this area had been known as Chemical Valley, as it contained the largest number of chemical plants in the United States. Coal and salt mining factories were well established in West Virginia as far back as the 1700s. However, Chemical Valley has its roots in World War I, when weapons were being produced for the war effort, and this continued on through the Vietnam era. In fact, during the Vietnam War, Agent Orange and other defoliants were produced in Chemical Valley.

It's likely that the magnitude of this leak could have been avoided. Weeks earlier, a few local residents reported a pungent smell they identified as "smelling like licorice" to the West Virginia Department of Environmental Protection. No preventative action was taken. On the day of the major spill, a number of complaints were filed, beginning at 7:30 am and continuing throughout the day. Many of the complaints were reported on social media, and some news agencies picked up the story. Still, no action was taken. The official "Do not use" water order was finally issued at 5:36 pm, with the exception of flushing the toilet [6]. That evening, the governor, Earl Ray Tomblin, went on the local news to caution that the water was not safe for "drinking, cooking, washing or bathing" [7]. Information about the spill was inadequately disseminated. Many didn't know about the spill until they watched the evening news, and some people hadn't seen the news or have access to this information.

The governor's order forced the closure of approximately 2,000 water-dependent facilities, 200 schools and many businesses [7]. The estimated cost to the local economy was \$19 million per day [8]. Federal Emergency Management Agency (FEMA) and the National Guard were sent to deliver water to the area, particularly to hospitals and nursing homes. However, this was a limited effort that did not benefit all the people affected by the spill. In addition to the struggle to find clean water to drink and for household use, many community markets and convenience stores inflated their prices; others had empty shelves.

In the following days, the public was urged to watch for "symptoms of exposure, including rashes, nausea, vomiting, and wheezing". In the immediate aftermath, the West Virginia Poison Center received over 1,900 calls, and over the ensuing three days after the spill, more than 200 people went to local hospital emergency rooms complaining of rashes, nausea, and other symptoms [5], [9].

On the fourth day, the governor held a press conference in which he announced that the risk to public health was decreasing, which was widely disbelieved. In fact, as the governor lifted the ban on the drinking water, emergency room visits rose for "skin rashes, eye irritation, nausea, vomiting, anxiety, and migraines" [5]. In their study the following year, Schade et al. [7], found that the days following the spill marked the largest acute illness outbreak caused by water contamination in United States history.

\section{LITERATURE REVIEW}

Across the Great Smoky Mountains from West Virginia, lies the US Marine Corps Base, Camp Lejeune. Much like Chemical Valley, it has a long history of using and manufacturing weapons. The base housed families, and from 1968 through 1985, three potent chemicals are known to have contaminated the water supply, trichloroethylene, tetrachloroethylene, and benzene. Families noticed health problems. Looking back at the health of mothers and children, two kinds of problems emerged among children, pre-term birth and being born at term, but having low birth weight; both of which can cause long lasting developmental 
problems [10]. The base in now what is called a Superfund site, an area identified as a risk to human health by the Environmental Protection Agency and eligible for the federal funding necessary for toxic clean-up. Anyone at Camp Lejeune who drank the water for 30 days or more, and has one or more of several kinds of cancer is entitled to free medical care for their treatment [11].

Travelling further South from Camp Lejeune is the Mississippi Delta region where Texas, Louisiana, Alabama and Florida form the Gulf of Mexico. On 20 April 2010, a British Petroleum oil well in the Gulf began to leak, and this became the biggest marine oil spill in history. The spill discharged about 5 million barrels of oil before the leak was capped 87 days later. It is one of the largest environmental disasters in the country's history, impacting the health of marine life, and the lives, which were nourished by fishing, both at the table and through the jobs it created. An environmental activist and mother, Marylee Orr stated, "It began for me with concern for the men on the rig, who would be alive and who wouldn't. And then it came from bayou wives who called to tell me their husbands were vomiting, had chest pains and nausea, headaches, and generally, were very sick". Her son Michael Orr said of the accumulation of disasters and the poverty of the region, "There's no real sense to me of bouncing back. It's clinging on" [12].

Perhaps important to how these events are now handled is a mother named Lois Gibbs. Love Canal was a 70 -acre (28 ha) suburban development near Niagara Falls, New York. The area had a long history of being a dumping ground for chemical waste. Beginning in the 1940s, Hooker Chemical Company used the land to dump 21,800 tons of chemical waste from the manufacturing of a variety of products, such as synthetic resins, dyes, perfumes, rubber, solvents and dioxin. In 1953, now owned by Occidental Chemical Corporation, the site was sold to the local school district. In the 1970s, Lois Gibbs was raising a young son with seizures and other inexplicable health issues. For years, people had wondered about a black fluid, seeping up through the ground, and in the canal. Community members complained about odors they couldn't identify that were on the school ground and elsewhere in the neighbourhood. It was Lois Gibbs' persistent attempts to alert elected officials that eventually led to biological sampling in 1978, which in turn led to the evacuation of hundreds of families. Borne out of her concerted efforts to be heard, President Jimmy Carter used emergency funds for a toxic disaster for the first time in the nation's history. The federal program, called Superfund, is now in place to identify and remediate places like Love Canal and Camp Lejeune [13]. The mothers of Woburn, Massachusetts and Hinkley, California spoke out to guide research towards toxins in their water, and the mothers in Wilmington, California are currently doing the same about their air quality. Despite these mothers' powerful voices, it remains a neglected area of public health and health research to more comprehensively, and strategically collect their concerns.

\section{AIMS OF THE STUDY}

Mothers and their children are often in the crucible of vulnerability. This is particularly true of disasters. Mothers may be pregnant and caring for children; some are caring for children and their own parents. In cases of divorce, mothers in the US are often the sole or primary care providers of children. This study focused on two things: one was to learn about the short and longer-term consequences of this disaster, as well as concerns of mothers through semistructured interviews who had gone through the West Virginia Water Crisis. These interviews were conducted in order to gain a more granular view and understanding of their experience of how they navigated risk, the actions they took during and after the disaster, and the adequacy of information they had. The interview also addressed how mothers made decisions about staying or leaving the area, and other thoughts about the area and their futures in it. 
The second goal was to begin the development of a semi-structured interview protocol that could be used in this disaster and potentially others. This would aid in the furthering of our combined knowledge of how different toxic disasters vary by chemical, by region, by the economic resilience of the community, and by the different ways in which the disaster is communicated, information imparted, risks mitigated, and follow-up put in place. This arose because toxic disasters are likely to increase in the coming years, as they become connected to severe weather events such as fires, hurricanes, and flooding, which are projected to increase in both frequency and intensity in the future.

\section{METHODS}

This project relied on qualitative methodology to explore mothers' experiences of the 2014 Freedom Industries spill in Chemical Valley, West Virginia. The creation of the initial semistructured interview protocol was a joint endeavor, and allowed both for the strategic collection of information, but also room to ask follow-up questions as needed. The idea was to begin the development of a semi-structured interview protocol that could be used in other disasters, thus allowing for cross-comparisons between different kinds of events by chemical, by age, gender, and location. Furthermore, the general history of these narratives is that they are often told to reporters. It is equally important and, perhaps, more so that they are also captured by researchers in healthcare. The semi-structured interview and protocol received IRB approval. The list of interview questions is provided in the Appendix.

Participants were recruited through social media and local newspaper ads to inform area residents of the study. To be included in the study, participants had to be a mother of a child 18 or younger, and living within the geographical zone affected during the toxic spill. The first 35 to meet this criterion were interviewed. The process of interviewing was a powerful emotional experience for those who participated; many reported being flooded with feelings they had during the time of the crisis. Unfortunately, some were unable to participate. One potential participant was in hospice care for cancer, and while she met the criteria, sadly failing health made it too difficult to participate. Another participant who endured a miscarriage during the spill found the idea of discussing her experience too emotionally challenging.

Throughout the interview, participants were asked to freely add to their answers and were offered the opportunity to contact the researcher if anything came to mind after the interview had taken place. This partnership created an opportunity to uncover ideas, thoughts, and questions that we, as researchers may not have considered. It also produced narratives that involved both the participant and researcher. It was thought that research need not only be the extraction of information, but itself part of a larger process of feeling greater control. All participants were told that what was learned from the project would be shared with them.

The interviewer for this project was born and raised in West Virginia. Rural communities have been known to be skeptical of outside researchers, particularly those that identify themselves as associated with mental health, which is often stigmatized in this region. It is likely that the interviewer's shared heritage with the mothers aided in creating a safe and comfortable space in which to share their stories.

All interviews were transcribed, and resulting transcripts were read, analyzed, and then coded with Quirkos software. The tapes, the transcriptions and the software allowed for the close analysis of text including content as well as the emotional overlay of the interviews. The themes generated were the effort of both authors. Much of the speech was in the vernacular of West Virginia, which does not come across as profoundly as in the mothers' voices themselves. 


\section{PARTICIPANT DEMOGRAPHICS}

Thirty-five mothers participated in this project. The mothers' ages ranged from 24-43. Of the participants, $45 \%$ were married, while $55 \%$ were either not married, separated, or divorced. $60 \%$ of participants had one or two children, and $40 \%$ had three or more children. Children ranged widely in age, with the majority (40\%) falling between 3 and 6 years of age. The mothers came from a diverse range of socio-economic backgrounds. $26 \%$ of the participants were stay at home mothers, while the other $74 \%$ came from varied occupations that included lawyers, social workers, educators, artists, government employees, and bankers. Educational backgrounds varied: $23 \%$ of participants had a high school diploma, $20 \%$ had a 4-year college degree, and 20\% possessed a master's degree and beyond, while another $20 \%$ reported having some college education. This group of participants likely have more years of formal education than is typical of West Virginia; the percentage graduating from college according to 2010 data, was $29.5 \%$ of people [14]. The vast majority $(86 \%)$ of participants reported having relatives living in the area. Most of those interviewed were white, which reflects the state's population at $93.34 \%$ Caucasian [15].

\section{RESULTS}

Nearly all the mothers spoke of deep ties to the area and strong feelings of being rooted in the land and culture of West Virginia. One mother remarked, "My generation, there's lots of family - I have 102 first cousins ... most of them still reside in Charleston". This connection to the area, and network of family ties was intricately linked to the emotional experience of those who went through the West Virginia Water Crisis. The following gives an overview of the mothers' thoughts and emotions, with special attention to those that might help in providing better health care, specifically mental health care.

All mothers were deeply impacted during and after the disaster. Most were afraid for their children at the time of the spill, and worried about their children's future health. Nearly all felt keenly disappointed with what they perceived was a delay in being notified, all the while describing the difficulty in accessing clean water for them and their families. Many conveyed a sense that there was little to no justice, particularly given that the company who was responsible has since gone bankrupt. Mothers were also burdened with the expectation of no help for emergent problems, particularly those that were health related. Several envisaged the environmental health of their children's generation as being worse and more challenging to mitigate in the future.

All participants were asked about their experience of the day they learned of the toxic spill. One mother described the smell of the water, "It smells like licorice, so that sort of made you think of food, and not something that could hurt you". Another mother said, "I remember ... I drove through town thinking something stinks, you know, like different than the normal chemical smell you have going through South Charleston". Describing the water coming out of her tap, one mother stated, "It was kind of just like a yellowish color, and then basically it just had like this very Listerine smell". She describes that non-essential workers were excused from work, and that black garbage bags were put over all the fountains, and they remained there for a year. One mother described doing the same in her home, to keep her daughter from touching the water, and perhaps to try and contain the fumes of the solvent as it evaporated. A pregnant woman dashed to the stores looking for bottled water, only to find empty shelves. She said of the water, "I think being pregnant for me was the scariest part, and how much had I taken in?" Another said she felt, "sheer desperate fear".

Some spoke of empty shelves, others recalled price gouging. One mother described it this way, "water became gold". A few left the state, but most stayed in the area. Mothers of sick 
children found themselves needing clean water, but unable to leave home to stand in the long lines to get clean water.

Not surprisingly, medical issues arose for many, in different ways across the generations. For a single mother whose baby was born at 652 grams, and who needed intensive home care, the scarcity of clean water for formula, and for sterilizing her baby's medical equipment, combined with her poverty, caused what she described as "a big depression". Not having a partner, she wasn't free to go out in search of clean water. One mother who has a son with asthma said that she had learned from others that, "when I flushed, I should not have the kids in the house". For one mother, there was the emergence of an unknown illness and high fever which needed hospitalization, and she needed to be evacuated to a hospital out of the area, as the local hospital couldn't spare the water to make the ice to keep her cool. The water caused rashes for some, and they were told to avoid contact. One mother said, "We all showered in it because we didn't have a choice, and we just weren't going to work dirty". Another participant said that her mother needed clean water for her CPAP breathing machine. Water was part of a lifeline for the sick and the well, as one woman put it, "Water is life".

While numerically not a large number of women, a few were pregnant at the time, and for them, the event was particularly difficult. One woman went into labor during the crisis, "We had a stillborn with our second (baby), and you know, who's to know if those things (chemicals) affected me. I mean, I don't think I even connected that until right now. She was already 12 inches long". One mother relayed that her son was born prematurely, requiring neo-natal intensive care, "He was born at 37 weeks and came out having breathing issues". Another mother noted, "My daughter was diagnosed in utero with a congenital heart defect. The correlation with that (chemical spill) has never been proven". Each of these women spoke about the distress in part stemming from not knowing. For one mother, pregnant at the time of the toxic spill, her child has since been diagnosed with autism. While she understood that there were several possible causes, she does come back to the toxic spill as a probable cause.

Worries about future health problems were also very common, "I was very, very scared for my baby. He had low immune system (functioning), anyway. I didn't know what he had been exposed to ... will we find out ten years down the road we were exposed to a cancercausing agent? And we knew from the get-go, nobody had our backs". One mother said, "I've known a lot of people that have passed. I don't know if it has anything to do with it, but have passed from cancer ... What's the future going to hold ... for my family?" Not only is there worry, but many also some spoke about a lack of responsibility, "I worry it's going to be one of those things where everybody develops like cancer, or like a brain tumor ... some kind of mystery health issue, and it's going to be very prevalent here - and then they're going to be like, oh it's not our fault, but then it is, you know?"

Compounding this future worry is the observation of one of the mothers, "We've seen a lot of doctors leave. We have to travel pretty far to get healthcare services that are specialized ... We just can't keep doctors here".

When the official water ban was lifted, one mother who was divorced talked about not wanting her child to use the tap water, but her ex-husband challenged her, not wanting their son going through life afraid. Four years later, a majority of participants still do not drink water from the tap. One woman said, "Currently, I cannot tell you that I trust the water source. We do bathe with the water, but I don't drink tap water. I don't prepare my food with tap water. I have zero faith that that situation was properly taken care of'.

When asked about leaving the area, some mothers reported that life would not be that much different outside of the state. One such mother grew up in Flint, Michigan, in which a problem of lead contamination in the municipal water supply was uncovered in 2014, the 
same year the present crisis occurred. She had moved to Charleston, West Virginia before the chemical spill occurred. As both Flint and Charleston have very similar median incomes, it's likely that economics played some role in the search for affordable housing. She noted: "I have it in my mind that it could just happen anywhere. If people in other states aren't having it happen to them right now, it could actually be happening to them, and they don't even realize it. Growing up in Flint, we never knew anything was wrong with our water, but come to find out there was something wrong the entire time. So it's almost as if you know, you're not really safe anywhere". For the majority of mothers, it was family ties that kept them from moving away. A mother described her family obligations: "I think I would stay here until something would happen with my parents. I feel an obligation to stay and take care of them. They are elderly. After that, I'm not sure that my ties to the mountain state will remain". Another mother said, "We're so rooted in this area, and this is where my family is. You know, my dad is older and sick, and he won't go anywhere. So, we're just kind of tied to the area". For a divorced mother, she couldn't imagine being able to take the children out of the state without a court battle.

Some did leave the area, and for those left behind, such as this mother, she recalls losing her network of friends, "the people that would have just came in my house and sat down...my entire girlfriend network left. They left because they could ... Within literally a year of the Water Crisis, I lost my community".

For some, this event has taken an economic toll. "I think financially we really put ourselves in a bind because of all the extra expenses. You don't think about bottled water, paper plates, paper towels. We bought adult washcloths, I mean, adult baby-wipes, things that you did not realize cost so much because you don't buy them that frequently". The Supplemental Assistance Program, or SNAP, helps low-income families buy food. One mother receiving the food allowance estimated that she used about "thirty to sixty dollars a month on water". While the toxic water spill eventually led to a lawsuit in which the plaintiffs won, with checks averaging several hundred dollars going out in September of 2018, it often didn't make up for the expenses that were incurred during the disaster, or the change in buying habits which ensued in the following years. A mother of three noted: "Show me some studies 15, 20, 30 years down the road and then elaborate that $\$ 400$ was sufficient for what happened to our state. But I suppose time will tell”.

Mothers talked about what they wanted other mothers to know, much of it having to do with being prepared. A mother with a sick child said she would caution mothers to have extras of all the necessities. Another mother said, "Rely on your gut, do everything you can from a mother's standpoint to protect your child for the future. No one else has your back. It's on you". This sentiment was echoed by another, "I hope it gets better. Don't rely on your government officials to take care of the situation for you". As far as chemicals and what is safe, one mother said there was no testing on what would constitute a safe level of a chemical on premature babies or infants so "to make sure that you're getting the most accurate and upto-date information". This sentiment summarized the feeling of needing to stay strong, "Just keep pushing through and it will get better eventually ... do what you got to do to survive".

Mothers were asked about what their child's generation would be contending with environmentally. Here almost all mothers felt the situation would worsen. Speaking about the environment, one mother said, "I would assume that my kids will have it much harder". While mothers throughout the interviews expressed love and concern for their children, at one point a mother said, "I am honestly scared for my children, and some days, I feel bad that we made the choice to have children". One of the causes of the despair was, "the regulations are loosening instead of tightening". As a consequence, a mother noted, "I believe that medical issues and illnesses are going to just continually worsen". Of her children's 
generation, one mother stated, "I hope they take better care of the resources, and of each other".

\section{DISCUSSION}

Prominent to many disasters which impact whole communities, including this one, is despair and anxiety about future health problems, and the fate of the land and all that it allows for the community, emotionally and economically. Health practitioners focus on single patients at a time, but what happens when the community is the patient? The mothers in this project voiced a wide range of concerns stemming from the spill. No one can know what health impacts there might be in the long run, or the fate of the Elk River, which flows through the cultural identity of the community, going back to when its sole inhabitants were Native Americans and elk roamed the banks.

In response to the crisis of the Deep Horizon oil spill, national research funding went to the Gulf Coast Health Alliance, a consortium that was in part university researchers, and also the community itself [16]. Community-based Participatory Research (CBPR), in which researchers partner with the community, to not only define the questions being asked, but to make sure that the perspectives of ordinary citizens are part of the data, is itself a way of treating a community. It addresses the mistrust and the feelings of being side-lined, while assuring that people's perspectives are incorporated into the findings. This research can also make sure that data is collected regarding the long-lasting health concerns that people worry about. Encouraging this form of citizen science is to acknowledge that the community has a role. Disasters take their toll on communities in part because for a moment in time, sometimes days, sometimes years, no one has a role that they once had, and one of the most prominent roles they do have, is that of victim.

Every mother who participated in this project interviewed with someone raised in West Virginia. Each person had the opportunity to revisit their interview and add things they felt were important to add. Each of them will get a synopsis of the project so that they understand that their stories were heard and are part of the story of their community. Healthcare's basic model is linked to the bio-psycho-social understanding of the individual. Additionally, to a large extent, the training of those in health care is disease or condition specific. With structured interviewing to help us learn more about major disasters, we hope that we can provide integrated care, which is event specific. It is hoped that these narratives help those in healthcare better understand this event and its long shadow.

From the research standpoint, we felt the need to develop a more systematized way of gathering information, so that we could further the knowledge of how they compare across events, places, and people.

There are several findings from this project relevant to future disaster management. On the largest level, the United States does not subscribe to the Precautionary Principle, the principle used throughout the EU and other places stating that a chemical or pharmaceutical must be proven to be safe, before it's introduced into public use. As it stands, currently in the US, people must demonstrate that they have been harmed for a chemical to be taken out of use. US industry uses more than 100,000 different chemicals, so the traditional scientific methodologies of public health find it increasingly difficult to establish whether any one chemical has harmed an ecosystem or negatively impacted human health [17]. This is because chemicals are released into an environmental context of other untested chemicals, or with other untested chemicals, and the synergistic effects produced when combined are unknown. Further, the focus of the spill was on two chemical compounds, MCHM and PPH, yet commercial products often have other ingredients, which go unexamined because they aren't considered active ingredients, and those too may have important health effects. 
The Hazard Communication Standard, which was revised in 2012, requires chemical manufacturers, or the distributor, to provide a 16-point Safety Data Sheet, formally known as the Material Safety Data Sheet. The current Safety Data Sheets are part of an international unified hazard communication system so that people using chemicals, and also first responders, can quickly understand the hazard potential and necessary first aid. Looking up the Safety Data Sheet for 4-Methylcyclohexanemethanol, this chemical is reported, "For Laboratory Use Only". First aid includes things that were not possible for those in the impacted area, such as "washing off with soap and water". Further, the first version of the Safety Data Sheet was issued on 26 March 2014, nearly four months after the spill [18].

Many mothers are worried about the long-term health effects to their children. Not only should we look at harm in the conventional ways of potentially causing cancers, but also focus on potential reproductive harm, epigenetic effects, and synergistic effects of chemicals as they combine with others during environmental releases. Of the small sample of 35 mothers, three reported reproductive problems.

This region lacked an appropriate disaster communication system that could have reached cell phones and landlines, or sounded warning alarms. Acting as its own notification system, part of the way that people knew something was wrong with the water was its smell and colour. There are of course numerous gasses and chemicals which are odorless, and if that was the case, it's conceivable that there would have been much more human exposure. It caused people to have burning in their eyes, respiratory issues, as well as nausea, so while little was known about the chemicals, the "Do not use" notification may have not only been slow in dissemination, but misleading. This was not only a toxic water disaster, but it was also an issue of toxic vapor, where exposure was occurring as it evaporated in toilets, where there is almost always standing water, and in the rivers and tributaries which were contaminated.

There has been a move in the US for several decades towards greater transparency. In 2004, the US National Library of Medicine and the Environmental Protection Agency created Toxmap. For those in disaster management, risk management, public health and medical research, this site has been invaluable. Selected industries, which are using an amount of a chemical thought to be potentially harmful to human or environmental health, are required to track their usages. The industries are mapped, and site-specific chemical use and waste is catalogued in a searchable format, which is accessible to the public. Toxic waste sites were also geospatially mapped. As it currently stands, the website reads, "TOXMAP website will be retired in the fall of 2019" [19]. Different municipalities and academic institutions may try and save the data, but importantly, it may not be kept up to date. In an era projected to have more natural disasters and, in turn, infrastructural damage, the loss of this knowledge is of great concern.

While it may go without saying, economics played a major role in this disaster, as it does in most. It could be considered a disaster within a disaster. These are often the most difficult to remediate in the long run. According to the 2018 Behavioral Risk Factor Surveillance System (BRFSS), 19\% of West Virginians live in poverty. More than a quarter, $26.3 \%$, consider their health fair to poor. West Virginia ranks the 3rd highest among the states in cancer prevalence [20]. Their current adult asthma prevalence as of 2017 is the 2 nd highest in the nation, with $12.7 \%$ of adults having asthma. Importantly for children, the infant mortality rate is $23 \%$ higher than for the US as a whole. Regarding mental health, the suicide rate is $27 \%$ higher than the national rate, according to the Appalachian Regional Commission's Health Disparities report [21]. It's likely reasonable to say that Chemical Valley had for years been caught between the need for chemical industry jobs, and perhaps the private desire for a healthier environment, so that lack of knowledge helped to ease the 
bind that many found themselves in. So in a disaster, it was harder to relay full information, plan evacuations, and work on appropriate disaster signalling in preparation for a toxic release.

While certainly there are limitations to this research, in that a granular view of a subset of people may not generalize to the whole population, it's hoped that it still provides an important range of challenges and obstacles, both in the short and long-term that mothers experienced.

In summary, better safety knowledge, more timely and accurate communication, and the preservation of systems of transparency are essential. As disasters are likely to increase in frequency and intensity, those in medical and mental health need to have educational training in meeting the needs of people, both during the crisis and in its long aftermath. Issues of water quality are numerous, and the long shadow that single events cast can last years, as this one illustrates. We look forward to using our protocol to examine other disasters in future years, to see the details of human experience that may help those in the frontlines of healthcare do the best they can.

\section{ACKNOWLEDGEMENT}

We would like to acknowledge Dr Patricia Wood for her substantial contribution to the ideas articulated in this text and for helping us guide this project forward.

\section{APPENDIX: INTERVIEW QUESTIONS}

1. How long have you lived in the area?

a. What are your historical/emotional ties to the area?

b. Before the spill happened - what would you say were your main concerns for your family and community?

2. What was it like to go through the chemical spill?
a. Remembering back to those days, what was your initial response to the spill?
b. Did your response change as time passed?
c. What were your main worries?
d. What were your worries for your family/children?
e. What are your current worries? How have they changed?
f. Have you gone through something similar in the past?

3. Can you speak a little about the aftermath of the spill, the days and weeks following?
a. How did you cope?
b. What if anything helped you to cope better?
c. What if anything made it harder to cope?
d. What resources, supports - if any - do you wish you had had to cope better?

4. Do you feel, having gone through the event, more prepared for other potential environmental disasters? Less prepared?

a. How did, or do you prepare? 
5. Do you see the rivers eventually getting cleaned up?

a. If yes: How would that happen? What would it take for that to happen?

b. If no: What will keep that from happening?

6. Do you see your child's generation also having to worry about the environmental things you do? Do you feel like their concerns might be the same as yours? How might they be different?

7. What do you think or feel are some of the biggest consequences of water pollution for you and your family?

8. Sometimes when people go through an environmental disaster, they think about moving. In your answering, there can be emotional factors, economic factors, or practical reasons; all of them are okay to talk about.

a. a. Did you think about moving?

b. What were some of the reasons you decided to stay?

c. What were some of the reasons you had considered moving?

9. In terms of this spill, do you feel there was accountability? Who is or was responsible?

10. Having gone through this, if you had a platform to talk to mothers across the country who are now facing disasters in their communities - what, if anything, might you want to say to them?

11. What are your hopes and dreams for your children?

12. Is there anything else you would like to say about this experience that you haven't mentioned?

\section{REFERENCES}

[1] Horzmann, K.A., de Perre, C., Lee, L.S., Whelton, A.J. \& Freeman, J.L., Comparative analytical and toxicological assessment of methylcyclohexanemethanol (MCHM mixtures associated with the Elk River chemical spill. Chemosphere, 188, pp. 599607, 2017.

[2] West Virginia Office of Miners' Health and Safety, Coal: West Virginia Coal Mining Facts. www.wvminesafety.org/wvcoalfacts.htm. Accessed on: 7 Jun. 2019.

[3] Cooper, W.J., Responding to crisis: The West Virginia chemical spill. Environmental Science and Technology, 48(6), 2014.

[4] Ward, D., West Virginia clean up. Aquosus Potentia, pp. 2-10, 2014.

[5] Osnos, E., Chemical Valley. The New Yorker. www.newyorker.com/magazine/2014/ 04/07/chemical-valley. Accessed on: 12 Aug. 2017.

[6] Whelton, A. et al., Residential tap water contamination following the Freedom Industries chemical spill: Perceptions, water quality, and health impacts. Division of Environmental and Ecological Engineering. https://pubs.acs.org/doi/suppl/10.1021/ es5040969/suppl_file/4es5040969_si_001.pdf. Accessed on: 31 May 2019.

[7] Schade, C.P., Wright, N., Gupta, $\bar{R}$., Latif, D.A. \& Jha, A., Self-reported household impacts of large-scale chemical contamination of the public water supply, Charleston, West Virginia, USA. PLoS One, pp. 1-14, 2015.

[8] Ginty, M., The waterborne toxic event: Why the West Virginia spill was not a freak occurrence. In These Times, 2014. 
[9] TOXNET: Toxicology Data Network, 4-Methyclcyclohexanemethanol. https://toxnet.nlm.nih.gov/cgi-bin/sis/search/a?dbs+hsdb:@term+@DOCNO+8182. Accessed on: 4 Jun. 2019.

[10] Ruckart, P., Bove, F. \& Maslia, M., Evaluation of contaminated drinking water and preterm birth, small for gestational age, and birth weight at Marine Corps Base Camp Lejeune, North Carolina: A cross-sectional study. Environmental Health, 13(99), 2014.

[11] US Department of Veterans Affairs, Camp Lejeune: Past water contamination. www.publichealth.va.gov/exposures/camp-lejeune/. Accessed on: 1 Jul. 2019.

[12] Sullivan, J. \& Rosenberg, B., I remember the mental chaos while they tried to seal the well and clean up the oil spill - How much fear and uncertainty everyone felt: An interview with Marylee and Michael Orr, Louisiana Environmental Action Network. New Solutions: A Journal of Environmental and Occupational Health Policy, 28(3), pp. 467-486, 2018.

[13] Gibbs, L.M., Excerpt from Love Canal: My story. Housewife's data. American Journal of Public Health, 101(9), pp. 79-82, 2011.

[14] US Department of Education, New state by state college attainment numbers show progress towards 2020 goal. www.ed.gov/news/press-releases/new-state-statecollege-attainment-numbers-show-progress-toward-2020-goal. Accessed on: 5 Jun. 2019.

[15] West Virginia Population 2019. www.worldpopulationreview/com/states/westvirginia-population. Accessed on: 5 Jun. 2019.

[16] Sullivan, J. et. al., Building and maintaining a citizen science network with fishermen and fishing communities post deepwater horizon oil disaster using a CBPR approach. New Solutions, 28(3), pp. 416-447, 2018.

[17] Henretig, F., Kirk, M. \& McKay, C., Hazardous chemical emergencies and poisonings. The New England Journal of Medicine, pp. 1638-1655, 2019.

[18] Eastman Chemical Company, Safety data sheet: Crude MCHM. www.msdsonline.com/sds-search/. Accessed on: 6 Jun. 2019.

[19] EPA's Toxic Release Inventory (TRI) and Superfund Programs. https://toxmap.nlm.nih.gov/toxmap/. Accessed on: 31 May 2019.

[20] West Virginia Department of Health and Human Resources, 2018 behavioral risk factor surveillance system. https://dhhr.wv.gov. Accessed on: 2 Jun. 2019.

[21] Appalachian Regional Commission. www.arc.gov. Accessed on: 5 Jun. 2019. 